\title{
Carotid Arterial Wall Dynamics During Gravity Changes on Partial-g Parabolic Flights
}

\author{
C. A. D. Leguy $\cdot$ P. Beck $\cdot$ P. Gauger • \\ L. E. J. Beck · U. Limper
}

Received: 28 October 2013 / Accepted: 14 July 2014 / Published online: 5 August 2014

(C) The Author(s) 2014. This article is published with open access at Springerlink.com

\begin{abstract}
The investigation of systemic blood pressure (BP) responses under partial-g conditions is of particular importance with respect to post-space-flight orthostatic intolerance. In this study, changes in vessel diameter and wall distension of the common carotid artery (CCA) were assessed under graded gravity. Measurements were performed on 8 healthy subjects in standing position under lunar $(0.16 \mathrm{~g})$, Martian $(0.38 \mathrm{~g}), 1.0 \mathrm{~g}$ and hypergravity $(1.8 \mathrm{~g})$ during partial-g parabolic flights. Data are reported as means \pm SE estimated by linear mixed effects modeling. The CCA diameter was significantly enlarged under Martian and lunar-g $(6.55 \pm 0.2$ and $6.54 \pm 0.2 \mathrm{~mm}$; $p<0.001$ each) with respect to $1.0 \mathrm{~g}(6.39 \pm 0.2 \mathrm{~mm})$. The CCA distension showed significant enlargement under Martian-g $(622 \pm 91 \mu \mathrm{m})$ with respect to $1.0 \mathrm{~g}(603 \pm 82$ $\mu \mathrm{m} ; \mathrm{p}<0.05)$. Furthermore, the distension was significantly lower under hyper-g with respect to $1.0 \mathrm{~g} \mathrm{(550 \pm 88}$ $\mu \mathrm{m} ; \mathrm{p}<0.001)$. These results show that rapid changes of gravitational stress induce significant modifications of hemodynamic parameters reflected in the CCA vessel wall diameter and distension. The increased vessel wall diameter under partial-g is likely due to the rise in mean BP at
\end{abstract}

C. A. D. Leguy $(\bowtie) \cdot$ P. Gauger · L. E. J. Beck

Institute of Aerospace Medicine, Department of Space Physiology, German Aerospace Center (DLR),

Linder Höhe, 51147 Cologne, Germany,

e-mail: carole.leguy@dlr.de

P. Beck

Department of General Surgery, University Witten/Herdecke, Helios Hospital Wuppertal, Wuppertal, Germany

U. Limper

Department of Anaesthesiology and Intensive Care Medicine, Merheim Medical Center, Hospitals of Cologne, University of Witten/Herdecke, Cologne, Germany the CCA level caused by the absence of hydrostatic pressure and may trigger the baroreflex to maintain homeostatis. We can assume that the increase in distension during the partial-g phase originates from a larger stroke volume and enhanced BP reflections. Furthermore, this study demonstrates the reliability of functional high resolution vascular ultrasound technique during parabolic flights.

Keywords Ultrasound · Partial gravity · Arterial baroreceptor - Arterial wall distension - Vascular diameter

\section{Introduction}

Human body limitations will probably be the most important obstacle to future long-term space missions. Major cardiovascular adaptations to microgravity environment are loss of intravascular volume (Diedrich et al. 2007), changes in baroreceptor reflex sensitivity (Ertl et al. 2000), loss of cardiac muscle mass (Perhonen et al. 2001), a decrease in systemic vascular resistance, and changes in vessel wall properties together with a lack of noradrenaline level (Zhang 2013; Meck et al. 2004). The resulting deconditioning of the cardiovascular system induces post-spaceflight orthostatic intolerance which remains a major issue for astronauts. A profound understanding of the cardiovascular response to gravitational and microgravitational stress is needed to improve countermeasure programs. Cardiovascular responses to transitional gravitational load is a fundamental aspect of understanding orthostatic tolerance that can be studied during parabolic flights.

Parabolic flights create rapid changes in gravity load from microgravity to $1.8 \mathrm{~g}$ over periods in the order of 20 seconds. These transitions in orthostatic stress induce acute changes in hydrostatic pressure present in arterial 
and venous system, as they form columns of fluid. This hydrostatic pressure is defined as $P_{h}=\rho g h$, where $\rho$ is the density of blood, $g$ the acceleration (gravity) and $h$ the height of the column of fluid (vertical distance from the aortic valve). With respect to $1 \mathrm{~g}$, microgravity loads lead to headward redistribution of blood (Aubert et al. 2005). We hypothesize that the graded partial gravity induced in this study (Pletser et al. 2012) will lead to a graded blood shift. Furthermore, those sudden alterations of the hemodynamics state will affect blood pressure level and trigger blood pressure regulation mechanisms such as the baroreflex. These mechanisms were demonstrated during parabolic flights through an increase and a fast decrease in heart rate during hyper-g and microgravity phase, respectively (Liu et al. 2012; Beckers et al. 2003). It has been observed that for standing subjects, mean arterial pressure is lower during the micro-gravity phase whereas pulse pressure is higher (Liu et al. 2012); similar reactions are observed for sitting subjects to a lesser extent (Pump et al. 1999).

Assessment of changes in arterial wall diameter at the Common Carotid Artery (CCA) provide a good estimate of systemic blood pressure (BP) dynamics from a proximal location. Furthermore, measurement of the CCA diameter provides an indication of blood pressure regulation at the level of the carotid baroreceptors located at the CCA bifurcation. Ultrasound techniques, with high spatial and temporal resolution, are recognized as the gold standard in arterial wall dynamics measurement and can be used to assess mean diameter, diameter-waveform and intimamedia-thickness of the CCA using radio-frequency signal techniques (Meinders and Hoeks 2004; Brands et al. 1998; Leguy et al. 2010; Leguy et al. 2009).

The objective of this study was to evaluate the feasibility of ultrasound measurements in assessing changes in vessel diameter and wall distension of the CCA on parabolic flights. The study was performed during the $2^{\text {nd }}$ Joint European Partial-g Parabolic Flights conducted by the Novespace company in Bordeaux, France, and sponsored by the ESA (European Space Agency), CNES (Centre national d'études spatiales) and DLR (Deutsches Zentrum für Luftund Raumfahrt) (Pletser et al. 2012).

\section{Materials and Methods}

\section{Subjects}

Eight healthy test subjects took part in the study: 4 men, age $34 \pm 7,69 \pm 10 \mathrm{~kg}, 175 \pm 8 \mathrm{~cm}$, BMI $22 \pm 1$; 4 women: age $26 \pm 4,60 \pm 4 \mathrm{~kg}, 167 \pm 3 \mathrm{~cm}$, BMI $21 \pm 1$. The experimental protocol was approved by the Agence française de sécurité sanitaire des produits de santé, and subjects provided written informed consent before participating. Subjects received scopolamine-hydrobromide injected subcutaneously as anti-motion-sickness medication [175 $\mu \mathrm{g}$ (men), $125 \mu \mathrm{g}$ (women)].

Partial-g parabola

Data were obtained during parabolic flights performed on the Airbus A300 Zero-G aircraft on 4 consecutive flights, 2 subjects were assessed per flight. Each flight was comprised of 31 parabolas, 13 Martian $(0.38 \mathrm{~g})$, 12 lunar $(0.16 \mathrm{~g})$, and $60 \mathrm{~g}$ parabolas. Ultrasound measurements were performed during 3 lunar and 3 Martian non-consecutive parabolas in each subject. For this study, we considered 5 phases for each parabola $(1 \mathrm{~g}, 1.8 \mathrm{~g}$, partial-g, $1.8 \mathrm{~g}, 1 \mathrm{~g})$ as illustrated in Fig. 1.

\section{Study Design}

Two previously trained ultrasonographers performed the measurements, alternating between flights. Right-hand side CCA wall diameter and distension were recorded beat-tobeat in standing subjects. Measurements started $30 \mathrm{~s}$ before and ended $30 \mathrm{~s}$ after the parabola maneuver. The ultrasonographer was standing behind the subject in a position that allowed watching the ultrasound scanner screen and minimized movements of the subjects to improve measurement quality (Fig. 2).

Beat-to-beat assessment of CCA wall diameter and distension were recorded using a Mylab ${ }^{\mathrm{tm}}$ One ultrasound scanner, a full 12 inch touch-screen display system equipped with a $12 \mathrm{MHz}$ linear array (Esaote Europe BV, Maastricht, The Netherlands). The device was used with cardiovascular settings that enable estimation of vessel wall mean diameter and distension, based on radio-frequency signal analysis as described by Brands et al. (Brands et al. 1999). The average diameter and distension are automatically reported for each heart cycle on the machine screen, see Fig. 3.

\section{Measurement Variability Analysis}

For the measured diameter $D$ and distension $\Delta D$, beat-tobeat variability $\sigma_{b}$, inter parabola variability $\sigma_{p}$ and inter subject variability $\sigma_{s}$ are derived for each phase. The estimates are based on the calculation of standard deviation as described by Leguy et al. 2009 .

\section{Statistical Analysis}

Linear mixed effect (LME) models fit by Restricted Maximum Likelihood estimations (REML) with g-load and gender as fixed effects and subject ID as random 


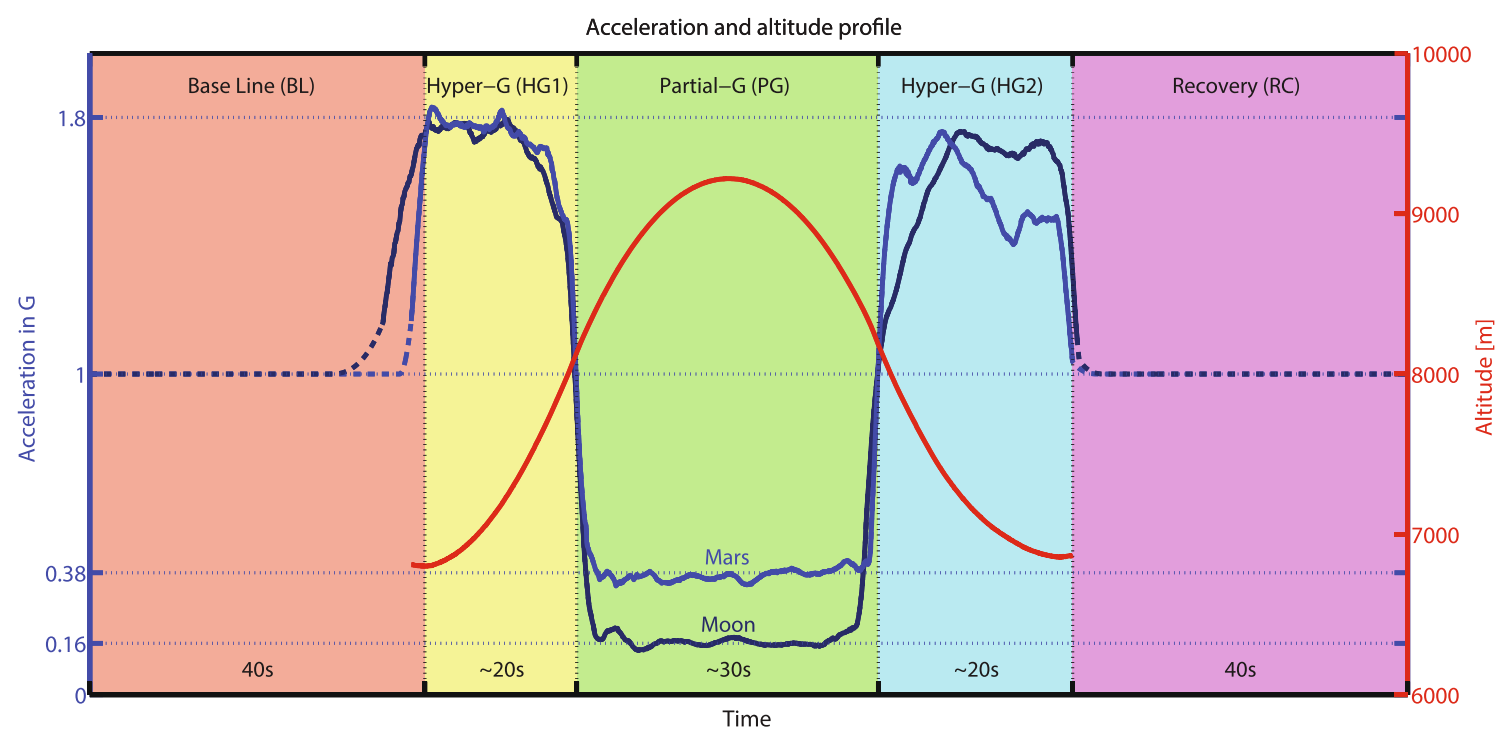

Fig. 1 G-profile for Moon and Mars parabola represented into 5 main phases (1g, $1.8 \mathrm{~g}$, partial-g, $1.8 \mathrm{~g}, 1 \mathrm{~g})$, and, the corresponding plane's altitude

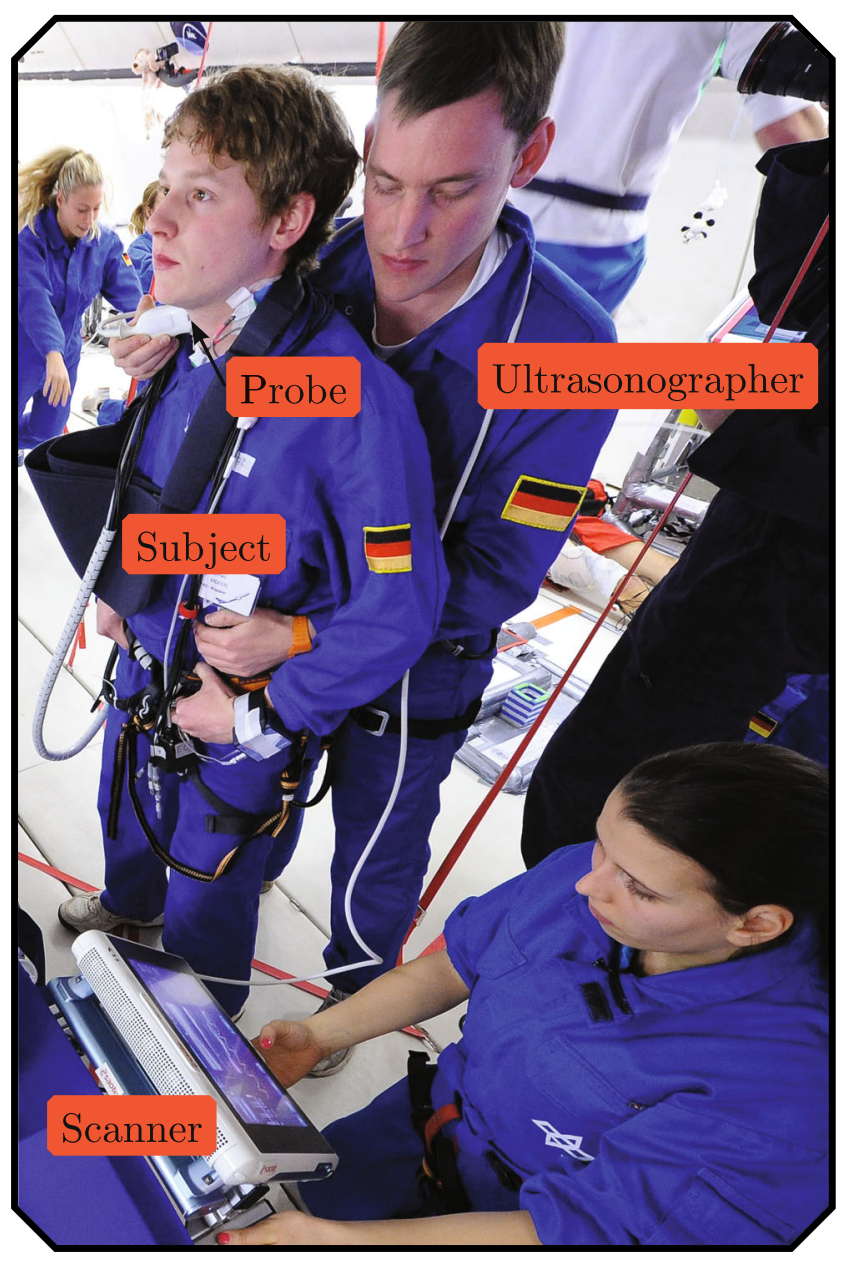

Fig. 2 In-flight imaging of the CCA on standing subject effect were constructed in order to assess g-load effects. The dependent variables were diameter or distension. Statistical analyses were carried out in IBM SPSS Statistics (IBM, Armonk, NY). Data are given as means and standard errors (SE). The level for statistical significance was set to $\alpha=0.05$.

\section{Results}

Repeated ultrasound assessments of the right common carotid artery (CCA) diameter and distension were obtained in eight healthy subjects during partial-g parabolas in standing body position with significant differences with respect to gravity load (Tables 1 and 2 a detailed response for one subject is depicted on Fig. 4).

\section{Diameter}

The modeled diameter of the right CCA, by LME, was significantly increased in partial-g with respect to $1.0 \mathrm{~g}$, whereas it was significantly smaller in hyper-g $(0.16 \mathrm{~g}$, $6.54 \pm 0.18 \mathrm{~mm}, p<0.001 ; 0.38 g, 6.55 \pm 0.19 \mathrm{~mm}, \mathrm{p}<$ $0.001 ; 1.0 \mathrm{~g}, 6.39 \pm 0.16 \mathrm{~mm} ; 1.8 \mathrm{~g}, 6.34 \pm 0.18 \mathrm{~mm}, \mathrm{p}=$ 0.017) (Fig. 5).

\section{Distension}

With respect to diamter, the modeled distension of the right CCA showed a likewise response during the parabolic flight maneuvers. Distension was significantly increased in 


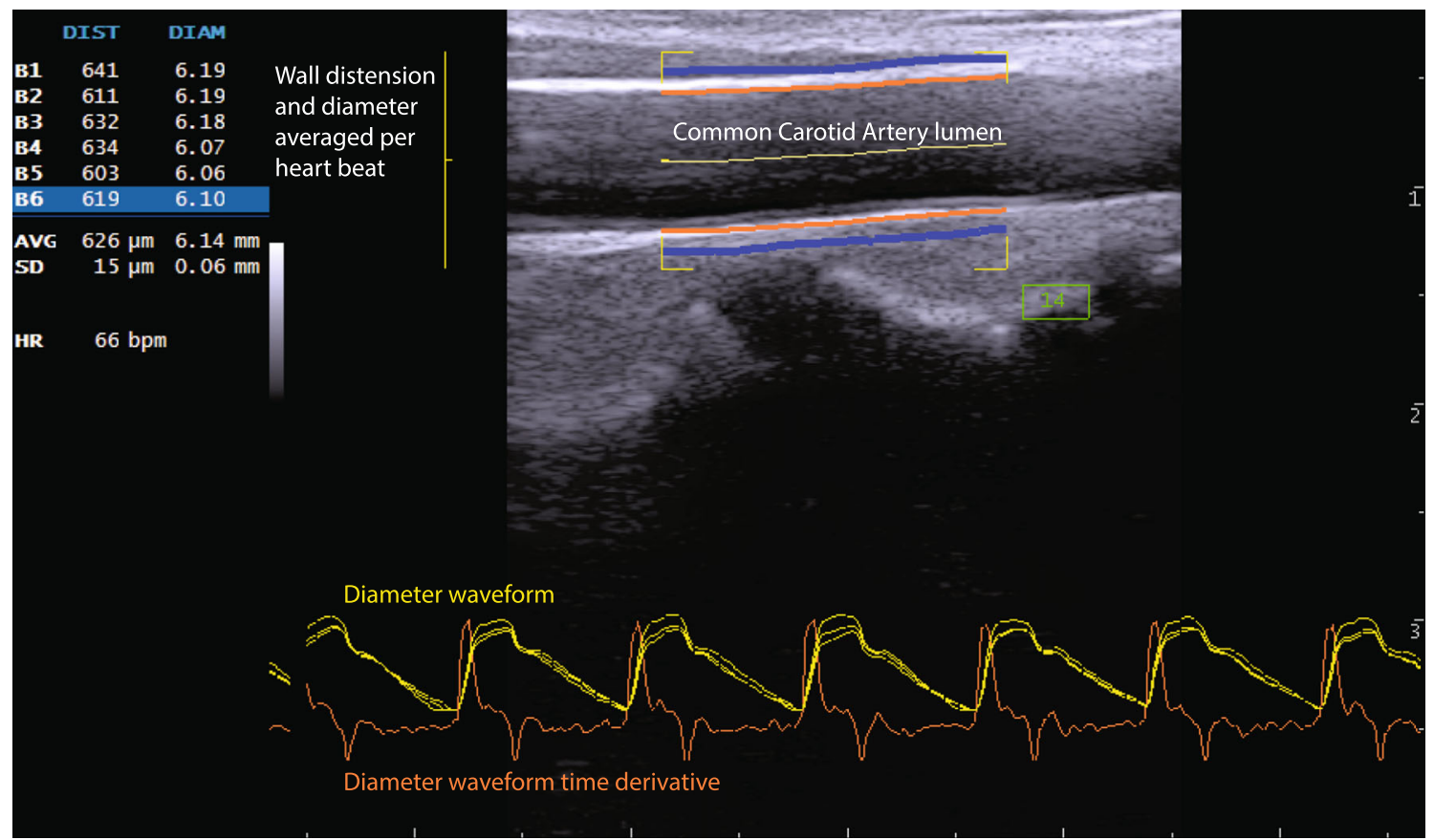

Fig. 3 Representative screenshot during an examination. Image of the CCA and detection of its vessel wall (orange lines) can be seen in the upper central part of the picture. Below vessel diameter (yellow) and

Martian- but not in lunar g, with respect to $1.0 \mathrm{~g}$, and it was significantly smaller in hyper-g $(0.16 \mathrm{~g}, 616 \pm 91 \mu \mathrm{m}, \mathrm{p}=$ $0.141 ; 0.38 \mathrm{~g}, 622 \pm 91 \mu \mathrm{m}, \mathrm{p}=0.043 ; 1.0 \mathrm{~g}, 603 \pm 82$ $\mu \mathrm{m} ; 1.8 \mathrm{~g}, 550 \pm 88 \mu \mathrm{m}, \mathrm{p}<0.01)$ Fig. 6.

\section{Discussion}

The study showed that cardiovascular ultrasound measurements are feasible in the challenging environment of gravity transitions in partial-g parabolic flight. We were able to demonstrate that diameter and distension of the CCA were all significantly affected by gravity changes. This is key to

Table 1 Diameter $D$ group average at the different phases: $1 \mathrm{~g}$ Baseline (BL), recovery (RC), hyper-g (1.8 g) and hyper-g and partial-g. For these phases, beat-to-beat variability $\sigma_{b}$, inter parabola variability $\sigma_{p}$ and inter subject variability $\sigma_{s}$ are shown. $\mathrm{N}$ is the number of assessed heartbeats

\begin{tabular}{llllll}
\hline & $D[\mathrm{~mm}]$ & $\sigma_{b}$ & $\sigma_{p}$ & $\sigma_{s}$ & $\mathrm{~N}$ \\
\hline $1 \mathrm{~g}(\mathrm{BL})$ & 6.43 & \pm 0.20 & \pm 0.30 & \pm 0.36 & 862 \\
$1.8 \mathrm{~g}$ & 6.34 & \pm 0.18 & \pm 0.32 & \pm 0.28 & 476 \\
Partial-g & 6.52 & \pm 018 & \pm 0.35 & \pm 0.37 & 764 \\
1.8 g & 6.35 & \pm 0.20 & \pm 0.32 & \pm 0.26 & 678 \\
1 g (RC) & 6.40 & \pm 0.12 & \pm 0.35 & \pm 0.27 & 372 \\
Average & 6.41 & \pm 0.18 & \pm 0.31 & \pm 0.31 & 630 \\
\hline
\end{tabular}

the first derivative of the diameter (orange) are shown beat-to-beat. In the upper left corner numerical results of distension and diameter are given for each heartbeat

our understanding of the adaptation of the human cardiovascular system to changing orthostatic stress.

Our findings of an increase in CCA diameter and of the CCA distensibility in partial-g with respect to initial $1 \mathrm{~g}$ values are within expected values, as they follow the behaviour of the mean blood pressure (MAP) of the CCA and the stroke volume (SV) of the heart.

These results present important differences to CCA measurements performed by Pannier et al. on 2 sitting subjects during 0-g parabolas (Pannier et al. 1995). While both groups observed increased CCA diameter during 0-g phase, Pannier et al. did not find increased vessel wall distensibility. These differences may result from the fact that their limited number of subjects was admittedly not sufficient for

Table 2 Distension $\Delta D$ group average at the different phases: $1 \mathrm{~g}$ Baseline (BL), recovery (RC), hyper-g (1.8 g) and partial-g. For these phases, beat-to-beat variability $\sigma_{b}$, inter parabola variability $\sigma_{p}$ and inter subject variability $\sigma_{s}$ are shown. $\mathrm{N}$ is the number of assessed heartbeats

\begin{tabular}{llllll}
\hline & $\Delta D[\mu \mathrm{m}]$ & $\sigma_{b}$ & $\sigma_{p}$ & $\sigma_{s}$ & $\mathrm{~N}$ \\
\hline $1 \mathrm{~g}(\mathrm{BL})$ & 572 & \pm 70 & \pm 67 & \pm 141 & 703 \\
$1.8 \mathrm{~g}$ & 504 & \pm 65 & \pm 62 & \pm 144 & 377 \\
Partial-g & 618 & \pm 82 & \pm 36 & \pm 162 & 639 \\
1.8 g & 556 & \pm 85 & \pm 49 & \pm 116 & 544 \\
1 g (RC) & 568 & \pm 64 & \pm 105 & \pm 158 & 308 \\
Average & 564 & \pm 73 & \pm 64 & \pm 144 & 514 \\
\hline
\end{tabular}


Fig. 4 Diameter and wall distention of the CCA during a moon type parabola for a female subject (partial-g phase between 20.1 and $44.5 \mathrm{~s})$
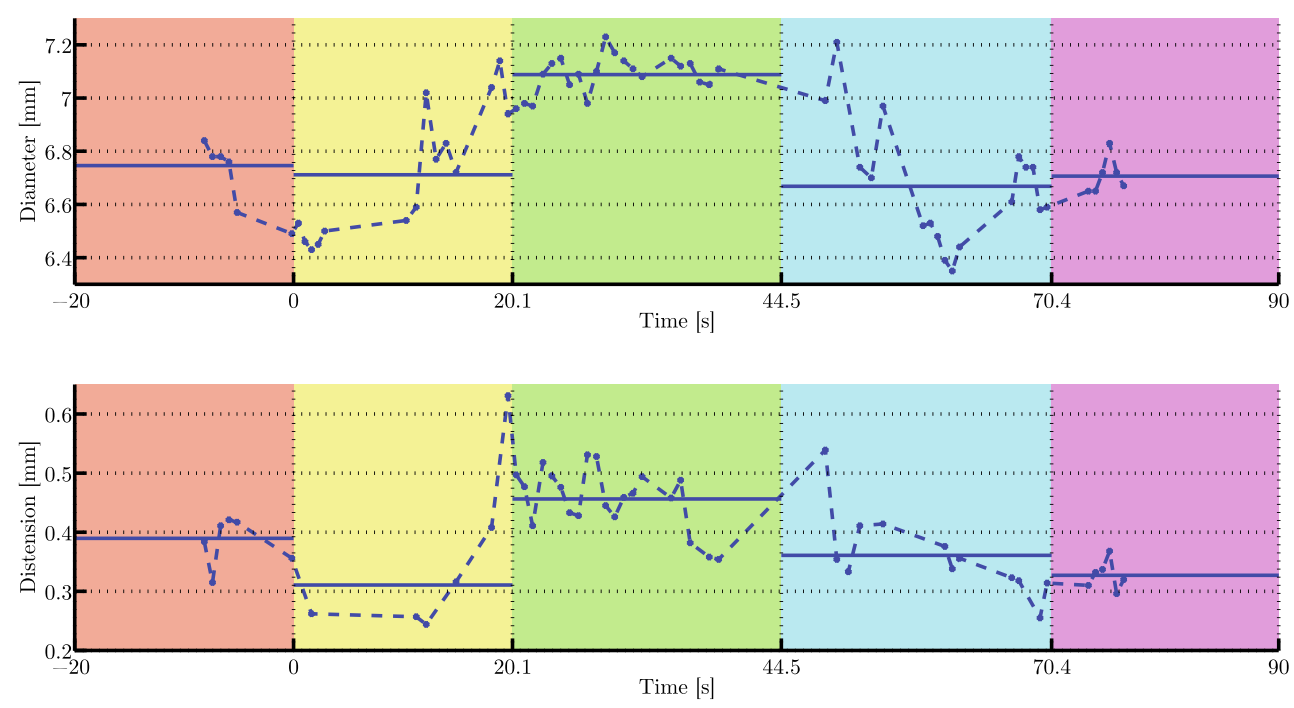

statistical analysis. Furthermore, in Pannier et al., the subjects were in sitting position whereas this study's subjects were standing.

From hyper-g to partial-g, the pressure difference due to the hydrostatic component between the CCA and the heart increases by roughly $20 \mathrm{mmHg}$. We measured an increase in diameter of $3 \%$ with respect to hyper-g phase. This diameter increase may trigger the baroreflex to maintain homeostatis and could be at the origin of the well recognized mean BP decrease during $0 \mathrm{~g}$ or partial-g phase of the parabola at the heart level (Aerts et al. 2012).

Experience and skill are required to obtain functional ultrasound measurements of good quality, such as the ones

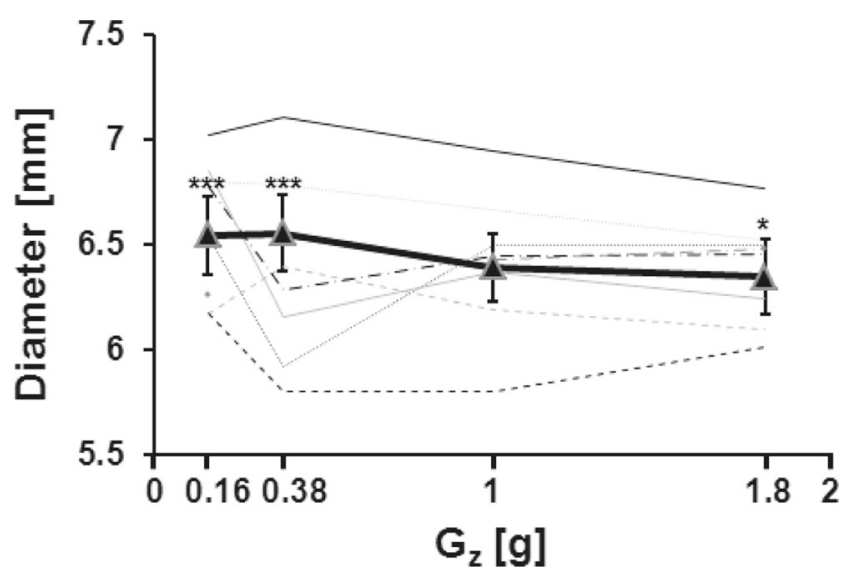

Fig. 5 The diameter of the right CCA by vascular ultrasound measurements in upright standing subjects during partial-g parabolic flight maneuvers is shown. The individual responses of the 8 subjects are presented by the thinner graphs. The bigger black continuous graph gives the modeled response by LME of all subjects taken together. Black triangles show the estimated diameters by LME in lunar $(0.16$ g) and Martian $(0.36 \mathrm{~g})$, Martian, 1.0 and $1.8 \mathrm{~g}$. Error bars represent SE. Asterisks give the significance level with respect to $1.0 \mathrm{~g}$ with $*<0.05 ; * *<0.01 ; * * *<0.001$ performed in this study. It also demands commitment from the subject. Parabolic flights are a very challenging environment due to spatial restriction, unstable positions (in the case of this study), changes in movement perception of the ultrasonographer (the arm feels heavier during hyperg) (Clément et al. 2009), and amplified subject movement during partial-g. As shown on Fig. 2, the ultrasonographer has to hold the subject to maintain their relative position. As such, a second operator was needed to take care of the ultrasound scanner operations while providing the ultrasonographer with visual feedback. Compared to values reported in literature, the beat-to-beat variability $\sigma_{b}$ is relatively high. For the diameter data, $\sigma_{b}$ is $0.18 \mathrm{~mm}$ whereas intra-registration variability of $0.06 \mathrm{~mm}$ was obtained from brachial artery measurements by Leguy et al. (Leguy et al. 2009) whilst the brachial artery being smaller and considered more difficult to assess. The higher variability may be

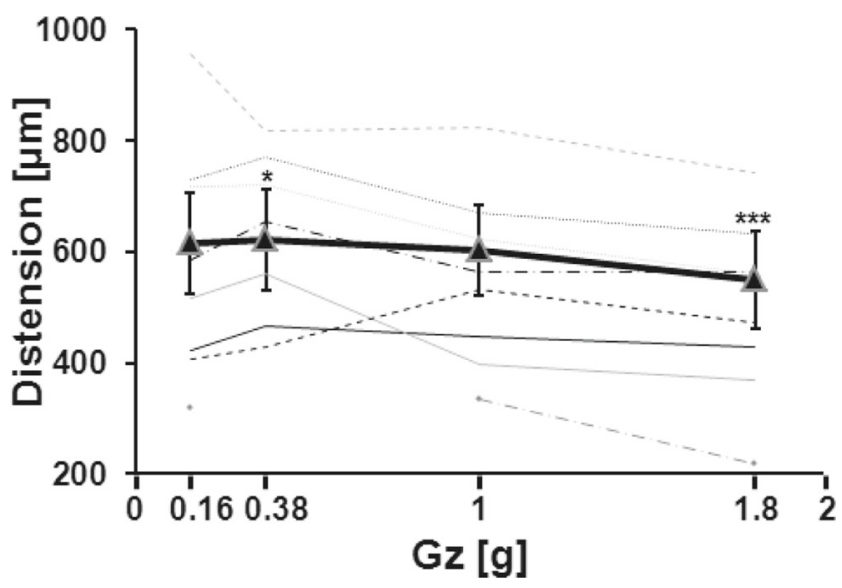

Fig. 6 The distension of the right CCA by vascular ultrasound measurements in upright standing subjects during partial-g parabolic flight maneuvers is shown. Figure characteristics are similar to that described for Fig. 5 
explained by the measurement conditions and transitional effects on physiological states within the defined parabolic phases. No direct comparison can be given for the beatto-beat variability of the distension since this one is given in percentage in Leguy et al.. The higher inter parabola variability $\sigma_{p}(0.31 \mathrm{~mm})$ may be explained by physiological state and measurement condition changes between the parabola (no similar data have been reported in literature to the author's knowledge). With regards to inter subject variability, a value of $0.91 \mathrm{~mm}$ have been reported by Paini et al. for the CCA diameter on 94 normotensive patients (Paini et al. 2006) which is 3 times higher than in this study. This difference may be explained by the group homogeneity (young and healthy subjects). Similarly, inter subject distension variability reported in this study is lower than the one reported by Paini et al., with $\pm 144 \mu \mathrm{m}$ and $\pm 262 \mu \mathrm{m}$, respectively. Ever though beat-to-beat variability found in this study are higher than the one reported in literature studies, the overall variability of the measurements remain acceptable and we have shown that such a set-up provides an adequate configuration for reliable measurements during parabolic flight.

The obtained variation of CCA diameter could have a significant effect on blood volume flow estimate. The observed changes in the order of $3 \%$ would induce a $6 \%$ error on blood volume flow estimate based on Doppler blood flow velocity when using an average baseline diameter. Contrary to blood volume flow estimate during physiological steady state, diameter variation of the CCA should be taken into account during parabolic flight. A simultaneous measurement of arterial diameter and blood flow velocity would improve blood volume flow estimation based on ultrasound Doppler.

This study shows that ultrasound allows the investigation of diameter regulation at the CCA level during lunar and Martian parabolic flights. The CCA of the young healthy subjects of this study were observed to be very adaptive to the cardiovascular stress of parabolic flight, suggesting sufficient and rapid blood pressure adaptation via the baroreflex. Future research will contribute to improve the method and to the investigation of elderly subjects with stiffer CCA to increase understanding of the changes of BP control with age.

Acknowledgments We are grateful to the test subjects for their participation in this study. The authors wish to thank the German Aerospace Center (DLR) for providing the flight opportunity during the $2^{\text {nd }}$ joint Parabolic Flight Campaign. The work herein was performed with the support of the Marie Curie International Outgoing fellowship of the European's $7^{\text {th }}$ Framework Programme for Research under contract number MC - IOF - 297967 and with the grant 50WB1255 of the German Federal Ministry of Economics and Technology.We are also thankful to Wolfram Sies for his technical support and to the appointees of NOVESPACE for all their skilful help.
We are furthermore grateful to Dr. Bernd Johannes for his statistical considerations.

Open Access This article is distributed under the terms of the Creative Commons Attribution License which permits any use, distribution, and reproduction in any medium, provided the original author(s) and the source are credited.

\section{References}

Diedrich, A., Paranjape, S.Y., Robertson, D.: Plasma and blood volume in space. Am. J. Med. Sci. 334, 80-5 (2007)

Ertl, A.C., Diedrich, A., Biaggioni, I.: Baroreflex dysfunction induced by microgravity: potential relevance to postflight orthostatic intolerance. Clin. Auton. Res. Off. J. Clin. Auton. Res. Soc. 10, 26977 (2000)

Perhonen, M.A., Franco, F., Lane, L.D., Buckey, J.C., Blomqvist, C.G., Zerwekh, J.E., Peshock, R.M., Weatherall, P.T., Levine, B.D.: Cardiac atrophy after bed rest and spaceflight. J. Appl. Physiol. 91, 645-653 (2001)

Zhang, L.-F.: Region-specific vascular remodeling and its prevention by artificial gravity in weightless environment, European journal of applied physiology (2013)

Meck, J.V., Waters, W.W., Ziegler, M.G., DeBlock, H.F., Mills, P.J., Robertson, D., Huang, P.L.: Mechanisms of postspaceflight orthostatic hypotension: low alpha1-adrenergic receptor responses before flight and central autonomic dysregulation postflight. Am. J. Physiol. Heart Circ. Physiol. 286, H1486-95 (2004)

Aubert, A.E., Beckers, F., Verheyden, B.: Cardiovascular function and basics of physiology in microgravity. Acta Cardiol. 60, 129-51 (2005)

Pletser, V., Winter, J., Duclos, F., Bret-Dibat, T., Friedrich, U., Clervoy, J.-F., Gharib, T., Gai, F., Minster, O., Sundblad, P.: The first joint european partial-g parabolic flight campaign at moon and mars gravity levels for science and exploration. Microgravity Sci. Tech. 24, 383-395 (2012)

Liu, J., Verheyden, B., Beckers, F., Aubert, A.E.: Haemodynamic adaptation during sudden gravity transitions. Eur. J. Appl. Phys. 112, 79-89 (2012)

Beckers, F., Seps, B., Ramaekers, D., Verheyden, B., Aubert, E.: Parasympathetic heart rate modulation during parabolic flights. Eur. J. Appl. Phys. 90, 83-91 (2003)

Pump, B., Videbaek, R., Gabrielsen, A., Norsk, P.: Arterial pressure in humans during weightlessness induced by parabolic flights. J. Appl. Phys. (Bethesda, Md.: 1985) 87, 928-32 (1999)

Meinders, J.M., Hoeks, A.P.G.: Simultaneous assessment of diameter and pressure waveforms in the carotid artery. Ultrasound Med. Biol. 30, 147-54 (2004)

Brands, P.J., Willigers, J.M., Ledoux, L.A., Reneman, R.S., Hoeks, A.P.: A noninvasive method to estimate pulse wave velocity in arteries locally by means of ultrasound. Ultrasound Med. Biol. 24, 1325-35 (1998)

Leguy, C.A.D., Bosboom, E.M.H., Gelderblom, H., Hoeks, A.P.G., van de Vosse, F.N.: Estimation of distributed arterial mechanical properties using a wave propagation model in a reverse way. Med. Eng. Phys. 32, 957-67 (2010)

Leguy, C.A.D., Bosboom, E.M.H., Hoeks, A.P.G., van de Vosse, F.N.: Model-based assessment of dynamic arterial blood volume flow from ultrasound measurements. Med. Biol. Eng. Comput. 47, 6418 (2009)

Brands, P.J., Hoeks, A.P., Willigers, J., Willekes, C., Reneman, R.S.: An integrated system for the non-invasive assessment of vessel wall and hemodynamic properties of large arteries by means of 
ultrasound. Eur. J. Ultrasound Off. J. Eur. Fed. Soc. Ultrasound Med. Biol 9, 257-66 (1999)

Pannier, B.M., Cuche, J.L., Benetos, A., Boutouyrie, P., Safar, M.E.: Carotidartery pulsatility during parabolic flights. Acta Astronaut. 36(8-12), 433-8 (1995)

Aerts, W., Joosen, P., Widjaja, D., Varon, C., Vandeput, S., Van Huffel, S., André, A.: Heart rate and blood pressure variability under moon, mars and zero gravity conditions during parabolic flights. Proceedings of the Joint Life Science Meeting 'Life in Space for Life on Earth'. Aberdeen, UK (2012)

Clément, G., Lathan, C., Lockerd, A., Bukley, A.: Mental representation of spatial cues in microgravity: writing and drawing tests. Acta Astronaut. 64(7), 678-681 (2009)

Paini, A., Boutouyrie, P., Calvet, D., Tropeano, A.-I., Laloux, B., Laurent, S.: Carotid and aortic stiffness: determinants of discrepancies. Hypertension 47, 371-6 (2006) 